\title{
LATENT ROOTS OF TRI-DIAGONAL MATRICES
}

\section{by F. M. ARSCOTT}

A considerable amount is known about the latent roots of matrices of the form

$$
L_{n} \equiv\left[\begin{array}{llllll}
b_{1} & c_{1} & & & & \\
a_{2} & b_{2} & c_{2} & & & \\
& a_{3} & b_{3} & c_{3} & & \\
& & \ldots \ldots \ldots \ldots \ldots . . & \\
& & & & a_{n} & b_{n}
\end{array}\right],
$$

in the case when each cross-product of non-diagonal elements, $a_{i} c_{i-1}$, is positive. One forms the sequence of polynomials $f_{r}(\lambda)=\left|L_{r}-\lambda I\right|$ for $r=1$, $2, \ldots n$, and observes that

$$
f_{r+1}(\lambda)=\left(b_{r+1}-\lambda\right) f_{r}(\lambda)-a_{r} c_{r-1} f_{r-1}(\lambda)
$$

then it is easy to deduce that (i) the zeros of $f_{n}(\lambda)$ and $f_{n-1}(\lambda)$ interlace-that is, between two consecutive zeros of either polynomial lies precisely one zero of the other (ii) at the zeros of $f_{n}(\lambda)$ the values of $f_{n-1}(\lambda)$ are alternately positive and negative, (iii) all the zeros of $f_{n}(\lambda)$-i.e. all the latent roots of $L_{n}$-are real and different.

For the corresponding matrix in which the cross-products of non-diagonal elements are negative, nothing can be said about the real nature of the roots, but it is possible to give some bounds for the real roots. There are also parallel theorems to (ii) and (iii) in a particular case-namely, when the diagonal elements are in increasing order of magnitude.

Theorem 1. Let $M_{n}$ denote the matrix

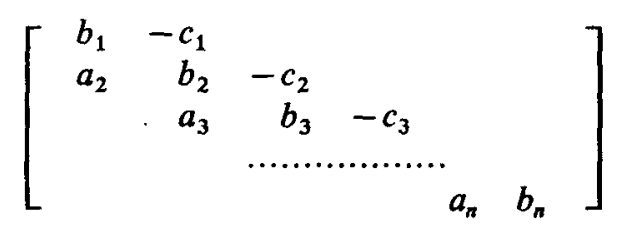

(all other elements being zero) in which all the $a, b, c$ are real, and each product $a_{i} c_{i-1}$ is positive. Then all the real latent roots of $M_{n}$ lie between the least and the greatest of the $b_{i}$.

Proof. We first establish a lemma. Consider the matrix $M_{n}^{*}$ obtained by replacing the diagonal elements in $M_{n}$ by elements $b_{i}^{*}$ which are all positive; we shall show that the real latent roots of this are all positive.

We observe that, because the $b_{i}^{*}$ and all the products $a_{i} c_{i-1}$ are all positive, 
every second-order principal minor in $M_{n}^{*}$ is positive. Every first-order principal minor is, of course, simply a $b^{*}$ and hence positive also. Now consider a principal minor of order $r$; expanding by its first row and column expresses it as the sum of positive multiples of two principal minors of orders $r-1, r-2$. Hence, inductively, all the principal minors of $M_{n}^{*}$ are positive (including $\left|M_{n}^{*}\right|$ itself).

Now consider the equation $f_{n}^{*}(\lambda) \equiv\left|M_{n}^{*}-\lambda I\right|=0$. Expanding the determinant according to its diagonal elements, we obtain

$$
f_{n}^{*}(\lambda)=\sum_{r}(-)^{n+r} d_{r} \lambda^{n-r}
$$

where all the $d_{r}$, being sums of principal minors of $M_{n}^{*}$, are positive. It is now obvious that no negative or zero value of $\lambda$ can make $f_{n}^{*}(\lambda)$ vanish, and the lemma is proved.

Now we return to the matrix $M_{n}$ and let $b_{l}$ be the least of the diagonal elements. The equation $\left|M_{n}-\lambda I\right|=0$ may be written

$$
\left|M_{n}-b_{l} I-\lambda^{\prime} I\right|=0, \text { where } \lambda^{\prime}=\lambda-b_{l},
$$

and since the matrix $\left(M_{n}-b_{l} I\right)$ has the form of the matrix $M_{n}^{*}$ considered in the lemma, all the real $\lambda^{\prime}$ satisfying (1) are positive-that is, all the real $\lambda$ are greater than $b_{l}$.

Finally, let $b_{g}$ be the greatest of the diagonal elements. The equation $\left|M_{n}-\lambda I\right|=0$ may be written

$$
\left|b_{g} I-M_{n}-\lambda^{\prime \prime} I\right|=0, \text { where } \lambda^{\prime \prime}=b_{g}-\lambda,
$$

and the matrix $\left(b_{g} I-M_{n}\right)$ has again the form of the matrix $M_{n}^{*}$ (the signs of the non-diagonal elements are reversed but the relevant products are unaltered). Thus every real $\lambda^{\prime \prime}$ satisfying (2) is positive-that is, all the $\lambda$ are less than $b_{g}$. All the $\lambda$ satisfying $\left|M_{n}-\lambda I\right|=0$, therefore, lie between $b_{l}$ and $b_{g}$, these values excluded.

Theorem 2. Let $M_{n}$ denote the matrix above, with the additional restriction imposed that $b_{1}<b_{2} \ldots<b_{n}$. Then the latent roots of $M_{n}$ and the latent roots of $M_{n-1}$ cannot interlace-in fact, between any two adjacent real latent roots of $M_{n}$ must lie, if any, an even number of real latent roots of $M_{n-1}$. If $f_{n}(\lambda)$ denote $\left|M_{n}-\lambda I\right|$, then at the zeros of $f_{n}(\lambda)$, the sign of $f_{n-1}(\lambda)$ is $(-)^{n-1}$ always.

Proof. Considering again the sequence of polynomials $f_{r}(\lambda) \equiv\left|M_{r}-\lambda I\right|$ $(r=1,2, \ldots n)$, we have

$$
\begin{aligned}
& f_{1}(\lambda)=b_{1}-\lambda \\
& f_{2}(\lambda)=\left(b_{1}-\lambda\right)\left(b_{2}-\lambda\right)+a_{2} c_{1} \\
& f_{r}(\lambda)=\left(b_{r}-\lambda\right) f_{r-1}(\lambda)+a_{r} c_{r-1} f_{r-2}(\lambda), r=2,3, \ldots \lambda,
\end{aligned}
$$

the last relation being established by expanding $\left|M_{r}-\lambda I\right|$ according to its last row and column. 
For large negative $\lambda$, all the $f_{r}(\lambda)$ are positive; for large positive $\lambda, f_{r}(\lambda)$ has the sign $(-)^{r}$; for brevity, we call this the "proper" sign of $f_{r}(\lambda)$ and $(-)^{r+1}$ the " reversed" sign.

From Theorem 1 it follows at once that for $\lambda \geqq b_{r}, f_{r}(\lambda)$ has its proper sign (otherwise it would have a zero in $\left(b_{r}, \infty\right)$ ). Let us suppose that, if possible, there is a value $\lambda_{0}$ of $\lambda$ such that $f_{n}\left(\lambda_{0}\right)=0$ and $f_{n-1}\left(\lambda_{0}\right)$ has its reversed sign. $\lambda_{0}$ must lie in $\left(b_{1}, b_{n}\right)$, and since $b_{1}<b_{2} \ldots<b_{n}$, there will be some $s$ such that $\lambda_{0}$ lies in the interval $b_{s-1} \leqq \lambda_{0}<b_{s}$. Then relation (3c) with $r=n$ shows that at $\lambda=\lambda_{0}, f_{n-2}\left(\lambda_{0}\right)$ also has its reversed sign; applying (3c) successively with $r=n-1, n-2, \ldots, s$ shows that all the $f_{\mathbf{r}}(\lambda)$ from $r=n-1$ down to and including $r=s-2$ have reversed signs at $\lambda=\lambda_{0}$. This, however, is impossible since $f_{s-1}(\lambda)$ and $f_{s-2}(\lambda)$ certainly have their proper signs when $\lambda \geqq b_{s-1}$, so the supposition is false and there can be no value of $\lambda$ where $f_{n}(\lambda)=0$ and $f_{n-1}(\lambda)$ has reversed sign.

It is easy to see, moreover, that if $f_{n}\left(\lambda_{0}\right)=0$, then $f_{n-1}\left(\lambda_{0}\right) \neq 0$; for if this were not so, then (from (3)) $f_{n-2}\left(\lambda_{0}\right)=f_{n-3}\left(\lambda_{0}\right)=\ldots=f_{1}\left(\lambda_{0}\right)=0$ also, hence $\lambda_{0}=b_{1}$, which contradicts Theorem 1. At every real zero of $f_{n}(\lambda)$, therefore, $f_{n-1}(\lambda)$ must have its proper sign, namely $(-)^{n-1}$.

The property of the latent roots of $M_{n}$ and $M_{n-1}$ follows immediately, and the theorem is proved.

I am grateful to a referee for some comments which shortened the proof of Theorem 1 .

Battersea College of Technology

LoNDON, S.W.11 